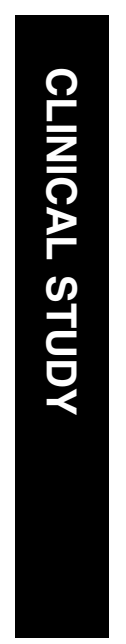

\title{
A three-year prospective, randomized and open comparison between latanoprost and timolol in Japanese normal-tension glaucoma patients
}

${ }^{1}$ Department of Ophthalmology The University of Tokyo Graduate School of Medicine

Tokyo, Japan

${ }^{2}$ Department of Ophthalmology Gifu University School of Medicine

Gifu, Japan

${ }^{3}$ Department of Ophthalmology Yamanashi University School of Medicine

Yamanashi, Japan

Correspondence:

M Araie, MD, PhD

Department of

Ophthalmology

The University of Tokyo

School of Medicine

7-3-1 Hongo, Bunkyo-ku

Tokyo 113-8655, Japan

Tel: $+81-3-3815-5411$

(\#36350)

Fax: + 81-3-3817-0798

E-mail: araie-tky@umin.ac.jp

Received: 9 April 2003 Accepted: 5 November 2003

Published online: 19 March 2004

The authors have no proprietary interest in the development or marketing of any product mentioned in the article.

\begin{abstract}
Purpose To compare the longitudinal effects of treatment on intraocular pressure (IOP) and visual field performance in Japanese normaltension glaucoma (NTG) between latanoprost and timolol.

Patients and methods This is an open-label, randomized, study. A total of 62 NTG patients were prospectively, consecutively enrolled. All study subjects were randomly assigned to $\mathbf{0 . 0 0 5 \%}$ latanoprost instillation once daily in the morning or $0.5 \%$ timolol instillation twice daily for a prospective 3-year follow-up, and underwent a routine ocular examination every month. Automated perimetry was performed every 6 months using Humphrey field analysers. Stereophotographs of optic discs were also obtained every 6 months.

Results Percentage of IOP reduction or the magnitude of IOP reduction showed no intergroup differences either at any time point (13-15\%). In the visual field, the estimated rate of change in the MD value (dB/year) was $-0.34 \pm 0.17$ (SE) for the latanoprost group, and $-0.10 \pm 0.18$ (SE) for the timolol group. The estimated rate of change in MD showed no significant difference from zero in both groups, and there were no statistical intergroup differences. No changes in the optic nerve head topography in the vertical cup-to-disc ratio and rim area measured by image-analysis techniques were observed in either group. There were no patients who dropped out due to the side effects of treatment regimens.
\end{abstract}

G Tomita1', M Araie'1, Y Kitazawa² and S Tsukahara ${ }^{3}$
Conclusion Both latanoprost and timolol single treatments reduced IOP by $13-15 \%$ at their trough effects for 3 years in Japanese NTG patients; both showed similar effects on visual field performance.

Eye (2004) 18, 984-989. doi:10.1038/sj.eye.6701373

Published online 19 March 2004

Keywords: normal-tension glaucoma; latanoprost; timolol; intraocular pressure

Normal-tension glaucoma (NTG) is a subgroup of open-angle glaucoma with a normal range of intraocular pressure (IOP) and without any other apparent causes for optic nerve head damage. According to recent epidemiological investigations, the prevalence of glaucoma with apparently normal IOP relative to open-angle glaucoma ranged from 32 to $66 \% .^{1-5}$ This is considerably higher than was expected. Nowadays, pathogenesis of NTG is considered multi-factorial. The risk factors for progression of NTG list IOP, ${ }^{6}$ presence of disc haemorrhage, ${ }^{7}$ larger area of peripapillary atrophy, ${ }^{8}$ myopia or myopic disc appearance, ${ }^{9}$ and lower blood pressure or peripheral vasospasm. ${ }^{10}$ IOP is the only treatable risk factor in the management of NTG at the time of investigation. The collaborative NTG study group reported that the surviving rate from progression of visual field defects was statistically significantly higher in the IOP reduction group than in the control group. ${ }^{11}$ However, the rate of cataract progression was significantly higher in patients in the IOP reduction group. About $70 \%$ of those who developed cataract had undergone filtering 
surgery to achieve sufficient IOP reduction. In Japanese NTG patients, of whom IOP $(16.2 \pm 1.8 \mathrm{mmHg})$ was surgically decreased to around $10-11 \mathrm{mmHg}$ over 6 years, ${ }^{12}$ the deterioration rate of visual field defects before surgery $(-1.05 \mathrm{~dB} /$ year in the Humphrey mean deviation) was also significantly reduced to $-0.44 \mathrm{~dB} /$ year after surgery. However, during the follow-up period, bleb-related infection was experienced in $8 \%$ of patients, and cataract progression was recognized in $13 \%$ of patients. Therefore, a treatment that has a strong IOPreducing effect with fewer complications is more desirable. For this reason, latanoprost may be the treatment of choice for NTG patients, because latanoprost reportedly has a potent hypotensive effect in NTG, ${ }^{13,14}$ has a more beneficial effect on ocular perfusion pressure than timolol, ${ }^{15}$ and has a beneficial effect on pulsatile ocular blood flow. ${ }^{16}$ To the authors' best knowledge, there are no previous studies on the longterm comparison of effects between timolol and latanoprost in normal-tension glaucoma patients.

The purpose of this prospective, randomized, open, multicentre study is to compare the longitudinal effects of treatment on IOP and visual field performance in Japanese NTG patients between latanoprost and timolol.

\section{Patients and methods}

\section{Patient enrolment and follow-up methods}

A total of 62 NTG patients were prospectively, consecutively enrolled for this study from July 1995 to March 1997 in the outpatient service in the hospitals of the University of Tokyo Graduate School of Medicine, Gifu University School of Medicine, and Yamanashi Medical College. NTG diagnosis was made for eyes with all of the following: (1) an untreated peak IOP of $\leq 21 \mathrm{mmHg}$ during follow-up, including that over a 24-h period (IOP measured every $2 \mathrm{~h}$ ) in both eyes; (2) the normal open anterior chamber angle; (3) the presence of glaucomatous optic nerve head change and corresponding visual field changes; (4) no ocular, rhinologic, neurologic, or systemic disorders responsible for optic nerve damage; (5) no history of haemodynamic crisis or previous ocular surgery or laser treatment in either eye. Further, patients enrolled with NTG eyes fulfiled the best-corrected visual acuity of $\geqslant 15 / 20$ with no media opacities, and their refractive errors in spherical equivalent did not exceed -8 or $+6 \mathrm{D}$. In addition, the mean deviation (MD) of the Humphrey visual field did not exceed $-12.0 \mathrm{~dB}$. The definitions of abnormal visual field measured by Humphrey perimetry in the current study were if patients had at least: (1) three adjacent test points (excluding the outermost rim) having a sensitivity lower than that of age-matched controls by
$5 \mathrm{~dB}$ or more; and/or (2) two adjacent points having a sensitivity lower than that of the controls by $10 \mathrm{~dB}$ or greater; and/or (3) a $10 \mathrm{~dB}$ difference across the nasal horizontal meridian in two adjacent points. If both eyes met the inclusion criteria, the worse eye in terms of the MD value of Humphrey visual field was selected for further analyses.

After obtaining written informed consent from all study subjects, patients were randomly assigned using the computer-generated randomization list kept in a sealed envelope to $0.005 \%$ latanoprost instillation once daily or $0.5 \%$ timolol instillation twice daily in both eyes for a prospective 3-year open follow-up. Patients who had already been treated with a topical ocular hypotensive drug discontinued treatment with that drug for at least 4 weeks before baseline measurements. Baseline measurements of IOP and reliable visual field were carried out twice within 12 weeks before starting eye-drop instillation. The average of the two measurements was adopted as the baseline IOP or visual field. After enrolment, patients underwent a routine ocular examination every month since initiation of the study, which included visual acuity testing, slit-lamp biomicroscopy, Goldmann applanation tonometry, and funduscopy. Patients were asked to instill one drop of $0.005 \%$ latanoprost into both eyes at $0900 \mathrm{~h}$ (Latanoprost group) or one drop of $0.5 \%$ timolol into both eyes at 0900 and $2100 \mathrm{~h}$ (Timolol group). At each visit, IOP was measured at $0900 \mathrm{~h}$ before the morning dose and compliance was checked. Automated perimetry was performed every 6 months using Humphrey field analysers with the program central 30-2, standard, fullthreshold strategy (Carl Zeiss Meditec, Dublin, CA, USA). All patients had reliable visual field measurements with a fixation loss of less than $20 \%$, and a false-positive rate and a false-negative rate of less than $30 \%$. If the visual field result did not meet the reliability criteria above, or if a fatigue effect was suspected, the examination was repeated within 1 month. The slope of the MD value was evaluated by the glaucoma change probability program of the Statpac II for the Humphrey visual field, and, if it was significantly negative $(P<0.05)$ with at least four or more results, the medication used in the other group was added. Stereoscopic optic disc photographs by a simultaneous stereoscopic fundus camera (Topcon TRC-SS, Topcon Inc., Tokyo, Japan) with ASA 100, 36-mm film (Fuji chrome 100, Fuji Film Inc., Tokyo, Japan) was also obtained after mydriasis every 6 months. The stereophotographs were analysed topographically by an experienced operator in a masked manner, using a computerized three-dimensional imageanalysis system (Topcon IMAGEnet, Topcon Inc, Tokyo Japan). The principles and outline of the instrument are described elsewhere. ${ }^{17-20}$ The inner border of the whitish 
scleral ring is referred to as the disc edge. The examiner defined four points at the disc edge, and the computer then calculated an ellipse fitting these points. The ellipse was taken as the disc border. The disc cup was defined as being $150 \mu \mathrm{m}$ below the surface of the optic disc. The vertical cup to disc ratio and rim area $\left(\mathrm{mm}^{2}\right)$ were obtained. A modification of Littmann's method adapted for use with the nontelecentric fundus camera was used to correct the magnification of the central fundus, taking into account anterior corneal curvature, refraction, and axial length (A Sakurai, PhD, Topcon Inc, Tokyo, Japan, unpublished data and formula, 1988). The study protocol was approved by the Ethical Review Committee of the individual institutes, and adhered to the tenets of the Declaration of Helsinki.

\section{Statistical analysis}

Changes in the IOP and the optic nerve head parameters throughout the follow-up period inside each group were evaluated using ANOVA and a paired $t$-test. Repeatedmeasure ANOVA or an unpaired $t$-test was used to evaluate the difference between the groups. A $P$-value of less than 0.05 was considered to be statistically significant. According to the data in the study of Mishima et $a l,{ }^{21}$ where effect on the IOP in Japanese primary open-angle glaucoma or ocular hypertension patients was compared between latanoprost and timolol with 90 patients in each group, the overall intergroup difference in IOP of $1.09 \mathrm{mmHg}$ over a 3-year follow-up is detectable by repeated-measure ANOVA with $\alpha=0.05$, and $(1-\beta)=0.8$, with 30 patients in each group. In estimating the effects of treatment on the time course of visual field performance in MD, regression analysis with a linear mixed model (SAS Statistical Software; SAS Institute, Cary, NC, USA) was applied. In this model, the random effect is added to correct the intraindividual correlation of the data, ${ }^{22}$ and the value of the estimated slopes of change in MD in the latanoprost and timolol groups is given. According to the data obtained in the study of Koseki et al, ${ }^{23}$ where time changes in MD in Japanese normal-tension glaucoma patients were studied without any treatment or under treatment of oral brovincamine, intergroup difference in the rate of change in MD over a 3-year follow-up of $0.44 \mathrm{~dB} /$ year is detectable with $\alpha=0.05$, and $(1-\beta)=0.8$, with 30 patients in each group.

\section{Results}

The latanoprost group consisted of 31 eyes of 31 patients (14 men and 17 women). The mean \pm SD at baseline was $55.6 \pm 10.0$ years for age, $15.0 \pm 1.6 \mathrm{mmHg}$ for IOP, and $-6.0 \pm 2.1 \mathrm{~dB}$ for MD. The timolol group consisted of 31 eyes of 31 patients ( 15 men and 16 women). The mean \pm SD was $54.3 \pm 8.5$ years for age, $15.9 \pm 2.0 \mathrm{mmHg}$ for IOP, and $-5.9 \pm 2.3 \mathrm{~dB}$ for MD. There were no statistically significant intergroup differences in age or MD value $(P>0.1)$, but the baseline IOP was higher in the timolol group $(P=0.0546)$. In the latanoprost group, 20 out of 31 patients were followed throughout the 3-year study period per protocol, while three out of 31 patients received additional timolol according to the criteria above, and eight out of 31 patients dropped out during the study period for various reasons (withdrawal from the study on their own initiative or moving from the area). In the timolol group, 19 out of 31 patients were followed throughout the study period, while five out of 31 patients received additional latanoprost according to the criteria, and seven out of 31 patients dropped out during the study period. However, in both the groups, no patients dropped out due to local or systemic side effects of eye drops or intolerance of eye drops. Since the results were essentially the same whether analysis was carried out in the intent to treat or per protocol group, the results in the intent to treat groups are shown below.

At 4 weeks and 156 weeks after starting treatment, the IOP in the latanoprost and timolol groups was reduced to $13.0 \pm 2.2,12.9 \pm 2.6($ mean $\pm S D)$, and $13.6 \pm 2.3$,

$14.0 \mathrm{mmHg}$ (mean $\pm \mathrm{SD}$ ), respectively; the difference from the baseline was statistically significant in both groups $(P<0.0001$, Figure 1). Both latanoprost and timolol reduced the IOP by about $2.0 \mathrm{mmHg}$ over a 3-year follow-up period. Repeated-measure ANOVA revealed no statistically significant intergroup differences in the time course of IOP (Figure 1). The percentage of IOP reduction was $13-15 \%$, showing no intergroup differences either at any time point ( $t$-test or ANOVA) (Figure 2).

The mean $\pm \mathrm{SD}$ of the MD value at 3 years was $-6.3 \pm 3.2 \mathrm{~dB}$ in the latanoprost group and $-5.6 \pm 2.9 \mathrm{~dB}$ in the timolol group. No difference was observed in the visual field throughout the follow-up period in the MD

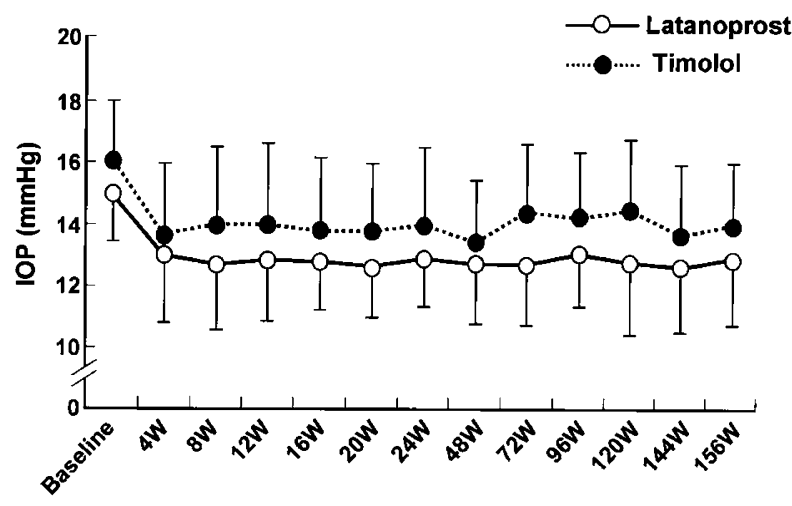

Figure 1 IOP changes in the latanoprost (open-circle) and the timolol (closed circle) treated groups. 


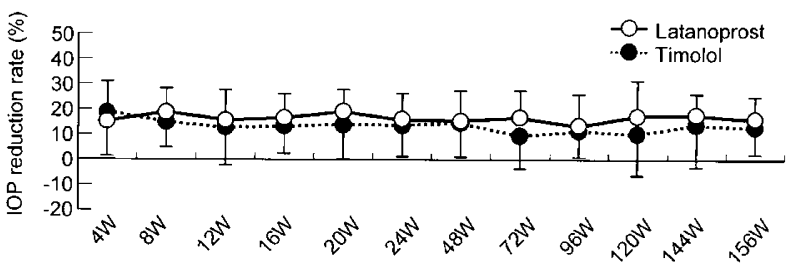

Figure 2 The percentage IOP reduction. Open circle: the latanoprost group. Closed circle: the timolol group.

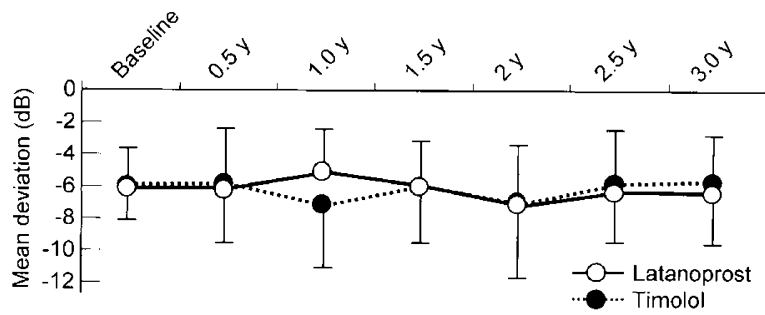

Figure 3 Changes in MD of the visual field. Open circle: the latanoprost group. Closed circle: the timolol group.

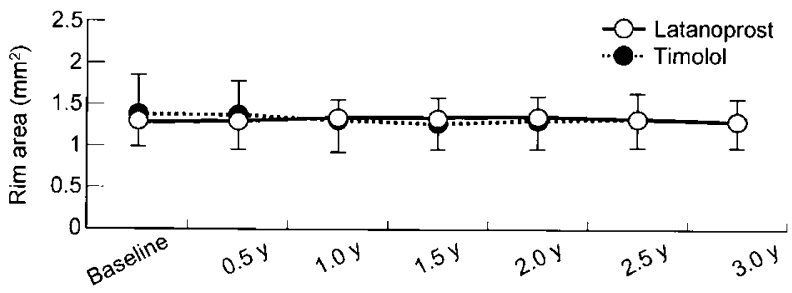

Figure 4 Changes in the rim area of the optic disc topography. Open circle: the latanoprost group. Closed circle: the timolol group.

value compared to the baseline in either group. In addition, no intergroup differences were observed throughout the study period (Figure 3). The estimated rate of change in the MD value ( $\mathrm{dB} /$ year) was $-0.34 \pm 0.17$ (SE) for the latanoprost group, and $-0.10 \pm 0.18$ (SE) for the timolol group. There were no statistically significant differences in the estimated rate of change in MD in the difference from zero in either group, and there were no statistically significant intergroup differences.

No changes in optic nerve head topography were observed in the vertical cup-to-disc ratio or the rim area in either group, and no significant intergroup difference was seen at any time point (Figure 4).

\section{Discussion}

This study followed up patients with NTG using latanoprost or timolol for 3 years. Both the latanoprost and timolol group showed similar effects on the IOP, a statistically significant reduction in IOP of about $2 \mathrm{mmHg}$ or $13-15 \%$ from the baseline over a 3 -year period as far as the trough effect is concerned. Latanoprost reportedly reduces IOP almost evenly by diurnal curve measurements in both primary open-angle glaucoma and NTG patients. ${ }^{13,15,24}$ It was also reported that timolol twice daily reduced IOP almost evenly in the morning, noon, and evening, after a 3-week treatment in NTG patients. ${ }^{15}$ Thus, it seems unlikely that the result significantly differs when IOP was also measured at other time points.

In this study, the overall intergroup difference over a 3-year follow-up in IOP of $1.09 \mathrm{mmHg}$ is detectable by a statistical power of $80 \%$. In addition, the decreasing sample size during the follow-up attenuated the strength of the equivalency finding. Therefore, it cannot be necessarily concluded that an IOP-reducing effect in Japanese NTG patients is exactly the same between timolol and latanoprost. However, between the groups, there were no differences in the rate of visual field progression in either the present follow-up period or method. In view of this point, it can be concluded that IOP difference, if any, between the latanoprost and the timolol groups in this study was not sufficient to be clinically significant.

Latanoprost, reportedly, has a greater effect on IOP reduction than the other glaucoma eye drops, ${ }^{21,25-27}$ although this claim is controversial. ${ }^{28}$ In NTG patients, Rulo $e a^{13}$ reported that the percentage reduction rate of IOP by $0.005 \%$ latanoprost once daily for 3 weeks was $21.3 \%$. Rulo et $a l^{13}$ and Tamada et $a l^{29}$ reported that, in patients with NTG, the magnitude of IOP reduction induced by latanoprost significantly correlates with the IOP level before treatment. That is, the higher the pretreatment pressure, the greater the IOP reduction. The present baseline IOP was around $15 \mathrm{mmHg}$, lower than that in the study of Rulo et al. This may partly explain the reason the percentage reduction rate of IOP by $0.005 \%$ latanoprost was between 13 and 15\% in the patients in this study. A similar explanation can be made for results of the timolol group, in which the IOP fall was lower than might be expected.

The estimated rate of change in the MD value $(\mathrm{dB} /$ year) was $-0.34 \pm 0.17$ (SE) for the latanoprost group, and $-0.10 \pm 0.18$ (SE) for the timolol group. This means that the MD value in the visual field showed only a small change in both groups in the 3-year study period. Koseki et $a l^{23}$ reported that, in their Japanese NTG patients who were followed without treatment, the estimated rate of change in MD was $-0.78 \mathrm{~dB} /$ year. Shigeeda $e t a l^{12}$ reported that, after trabeculectomy with antiproliferative agents in progressive Japanese NTG eyes, the rate of change in MD averaged $-0.44 \mathrm{~dB} /$ year. The results of the 
estimated rate of change in MD in the present study groups were very similar to the rate after trabeculectomy in progressive NTG eyes. In this study, the MD was the only parameter of visual field defects evaluated. However, it must be noted that change in MD is not sensitive to the local progression of visual field damage. The estimated rate of change of MD in the latanoprost group, $-0.34 \mathrm{~dB} /$ year, was of borderline significance $(P=0.053)$. The present findings suggest that visual field damage progresses slowly, at least some NTG eyes, at an IOP level of $13 \mathrm{mmHg}$. This is expected to almost halve the further progression in primary open-angle glaucoma eyes, ${ }^{30}$ and is comparable to the results of a report by the collaborative NTG study group ${ }_{1}^{11}$ in which it was noted even in patients who achieved a $30 \%$ reduction of IOP from the baseline, $20 \%$ of whom still showed a progression of visual field damage. A 3-year follow-up may not be long enough to detect any differences between the two treated groups, since, for example, an increase of the rate of developing cataract in patients treated with aqueous suppressant, such as timolol, has been reported..$^{31,32}$

In conclusion, both latanoprost and timolol single treatments for 3 years had a similar effect to each other on lowering IOP and visual field performance in Japanese normal-tension glaucoma patients. The single use of latanoprost or timolol was found to reduce IOP in NTG patients with a baseline IOP of $15 \mathrm{mmHg}$ by $2 \mathrm{mmHg}$ at its trough effect, without loss of effect for at least 3 years.

\section{References}

1 Mason RP, Kosoko O, Wilson MR, Martone JF, Cowan CL, Gear JC et al. National survey of the prevalence and risk factors of glaucoma in St. Lucia, West Indies. Part I. Prevalence findings. Ophthalmology 1989; 96: 1363-1368.

2 Shiose Y, Kitazawa Y, Tsukahara S, Akamatsu T, Mizokami $\mathrm{K}$, Futa $\mathrm{R}$ et al. Epidemiology of glaucoma in Japan-a nationwide glaucoma survey. Jpn J Ophthalmol 1991; 35: 133-155.

3 Tielsch JM, Katz J, Singh K, Quigley HA, Gottsch JD, Javitt J et al. A population-based evaluation of glaucoma screening: the Baltimore Eye Survey. Am J Epidemiol 1991; 134: 1102-1110.

4 Klein BEK, Klein R, Sponsel WE, Franke T, Cantor LB, Martone J et al. Prevalence of glaucoma. The Beaver Dam Eye Study. Ophthalmology 1992; 99: 1499-1504.

5 Dielemans I, Vingerling JR, Wolfs RCW, Hofman A, Grobbee DE, de Jong PTVM. The prevalence of primary open-angle glaucoma in a population-based study in the Netherlands. The Rotterdam Study. Ophthalmology 1994; 101: 1851-1855.

6 Crichton A, Drance SM, Douglas DD, Schulzer M. Unequal intraocular pressure and its relation to asymmetric visual field defects in low-tension glaucoma. Ophthalmology 1989; 96: 1312-1314.
7 Ishida K, Yamamoto T, Kitazawa Y. Risk factors associated with progression of normal-tension glaucoma. J Glaucoma. 1998; 7: 372-377.

8 Araie M, Sekine M, Suzuki Y, Koseki N. Factors contributing to the progression of visual field damage in eyes with normal-tension glaucoma. Ophthalmology 1994; 101: 1440-1444.

9 Mitchell P, Hourihan F, Sandbach J, Wang JJ. The relationship between glaucoma and myopia. The Blue Mountains Eye Study. Ophthalmology 1999; 106: 2010-2015.

10 Meyer JH, Brandi-Dohrn J, Func J. Twenty four hour blood pressure monitoring in normal tension glaucoma. $\mathrm{Br} J$ Ophthalmol 1996; 80: 864-867.

11 Collaborative Normal-Tension Glaucoma Study Group. Comparison of glaucomatous progression between untreated patients with normal-tension glaucoma and patients with therapeutically reduced intraocular pressure. Am J Ophthalmol 1998; 126: 487-497.

12 Shigeeda T, Tomidokoro A, Araie M, Koseki N, Yamamoto S. Long-term follow-up of visual field progression after trabeculectomy in progressive normal-tension glaucoma Ophthalmology 2002; 109: 766-770.

13 Rulo AH, Greve EL, Geijssen HC, Hoyng PF. Reduction of intraocular pressure with treatment of latanoprost once daily in patients with normal-pressure glaucoma. Ophthalmology 1996; 103: 1276-1282.

14 Waldock A, Snape J, Graham CM. Effects of glaucoma medications on the cardiorespiratory and intraocular pressure status of newly diagnosed glaucoma patients. Br J Ophthalmol 2000; 84: 710-713.

15 Drance SM, Crichton A, Mills RP. Comparison of the effect of latanoprost $0.005 \%$ and timolol $0.5 \%$ on the calculated ocular perfusion pressure in patients with normal-tension glaucoma. Am J Ophthalmol 1998; 125: 585-592.

16 McKibbin M, Menage MJ. The effect of once-daily latanoprost on intraocular pressure and pulsatile ocular blood flow in normal tension glaucoma. Eye 1999; 13: 31-34.

17 Varma R, Steinmann WC, Spaeth GL, Wilson RP. Variability in digital analysis of optic disc topography. Graefes Arch Clin Exp Ophthalmol 1988; 226: 435-442.

18 Varma R, Douglas GR, Steinmann WC, Wijsman K, Mawson D, Spaeth GL. A comparative evaluation of three methods of analyzing optic disc topography. Ophthalmic Surg 1989; 20: 813-819.

19 Nanba K, Shirakashi M, Fukuchi T, Iwata K. Stereophotometry of optic disc cupping with a computer image analyzer IMAGEnet. Rinsho Ganka 1989; 43: 535-538.

20 Shirakashi M, Nanba K, Iwata K. Changes in reversal of cupping in experimental glaucoma. Longitudinal study. Ophthalmology 1992; 99: 1104-1110.

21 Mishima HK, Masuda K, Kitazawa Y, Azuma I, Araie M. A comparison of latanoprost and timolol in primary open-angle glaucoma and ocular hypertension. A 12-week study. Arch Ophthalmol 1996; 114: 998-999.

22 Laird NM, Ware JH. Random effects models for longitudinal data. Biometrics 1982; 38: 963-974.

23 Koseki N, Araie M, Yamagami J, Shirato S, Yamamoto S. Effects of oral brovincamine on visual fields damage in patients with normal-tension glaucoma with low-normal intraocular pressure. J Glaucoma 1999; 8: 117-123.

24 Liu CJ-L, Ko Y-C, Cheng C-Y, Chiu AW, Chou JC, Hsu W-M et al. Changes in intraocular pressure and ocular perfusion pressure after latanoprost $0.005 \%$ or brimonidine tartrate $0.2 \%$ in normal-tension glaucoma patients. Ophthalmology 2002; 109: 2241-2247. 
25 Camras CB, Wax MB, Ritch R, Weinreb R, Robin AL, Higginbotham EJ et al. Latanoprost treatment for glaucoma: effects of treating for 1 year and of switching from timolol. United States Latanoprost Study Group. Am J Ophthalmol 1998; 126: 390-399.

26 Nordmann JP, Soderstrom M, Rouland JF, Malencaze F. Comparison of the intraocular pressure lowering effect of latanoprost and a fixed combination of timolol-pilocarpine eye drops in patients insufficiently controlled with beta adrenergic antagonists. French Latanoprost Study Group, and the Swedish Latanoprost Study Group. Br J Ophthalmol 2000; 84: 181-185.

27 Emmerich KH. Comparison of latanoprost monotherapy to dorzolamide combined with timolol in patients with glaucoma and ocular hypertension. A 3-month randomized study. Graefes Arch Exp Ophthalmol 2000; 238: 19-23.

28 Watson P, Stjernschantz J, the Latanoprost Study Group. A six-month, randomized, double-masked study comparing latanoprost with timolol in open-angle glaucoma and ocular hypertension. Ophthalmology 1996; 103: 126-137.

29 Tamada Y, Taniguchi T, Murase H, Yamamoto T, Kitazawa Y. Intraocular pressure-lowering efficacy of latanoprost in patients with normal-tension glaucoma or primary openangle glaucoma. J Ocul Pharmacol 2001; 17: 19-25.

30 The advanced glaucoma intervention study (AGIS): 7 . The relationship between control of intraocular pressure and visual field deterioration. The AGIS Investigators. Am J Ophthalmol 2000; 130: 429-440.

31 Heijl A, Leske MC, Bengtsson B, Hyman L, Bengtsson B, Hussein M. Early Manifest Glaucoma Trial Group.

Reduction of intraocular pressure and glaucoma progression: results from the early manifest glaucoma trial. Arch Ophthalmol 2002; 120: 1371-1372.

32 Leske MC, Wu SY, Nemeasure B, Hennis A, Barbados Eye Studies Group. Risk factors for incident nuclear opacities. Ophthalmology 2002; 109: 1303-1308. 